\title{
Zur linguistischen Sinnsuche in- und ausserhalb von schriftsprachlichen Korpora \\ Eine Replik auf Wolfgang Teuberts Beitrag
}

Raphael Berthele (Bern)

\begin{abstract}
In this reply to Wolfgang Teubert's contribution, some of the central tenets of his position are questioned. The primacy of literacy for linguistic investigations as advocated by Teubert is challenged, since the most important function of language is oral communication, and most of the world's languages exist exclusively in oral use. Counter-evidence to Teubert's claim according to which oral languages do not develop metalinguistic awareness (as e.g. the concept of "word") is given. The author scrutinizes the value added by Teubert's programmatic suggestion of "hermeneutic corpus linguistics" to the linguistic disciplines, since the sole focus on "discourse objects" unnecessarily narrows down the scope of linguistic investigation. Moreover, it seems to lead to backgrounding and neglect of what linguistics is all about: the comprehensive study of the structures, meaning potentials and usage patterns of the languages and varieties of the world, and the study of their usage-based emergence and evolution.
\end{abstract}

\section{Die Linguistik der diskursiv konstruierten Wirklichkeiten}

In seinem programmatischen Artikel in dieser Ausgabe von Linguistik online legt Wolfgang Teubert dar, was die zentrale Aufgabe der Linguistik in seinen Augen sei, und insbesondere inwiefern sie diese zentrale Aufgabe mit den Mitteln der Korpuslinguistik angehen kann und soll. Teubert vertritt dabei pointiert die Position, dass sich Sprachwissenschaft primär der Beschreibung und dem Vergleich von verschiedenen, parallel oder diachron unterschiedlichen "diskursiv konstruierten Wirklichkeiten" widmen soll. Die Beschreibung des Sprachsystems soll in den Hintergrund treten zugunsten einer "hermeneutischen Korpuslinguistik", die von den folgenden Grundannahmen ausgeht:

1. a) Orale Sprache ist kontextabhängig, immanent deiktisch, nicht abstrakt, sondern konkret.

b) Orale Kulturen/Gesellschaften haben keine metasprachlichen Diskurse, es fehlt das Bewusstsein der Symbolhaftigkeit sprachlicher Zeichen, sie kennen keine Linguistik, sie haben beispielsweise kein Konzept wie 'Wort'.

c) Oralität ist zwar sprachgeschichtlich primär, erst Schriftlichkeit aber schafft vom Kontext losgelöste "Diskursobjekte", auf die sich geschriebene Texte beziehen. 
d) Der Gegenstand (Daten) der Linguistik sind deshalb (fast) ausschliesslich geschriebene Texte.

2. a) Texte sind Rekombinationen, Umformungen, Neuinterpretationen von bereits bestehenden Texten.

b) Texte haben eine Bedeutung, die sich losgelöst von ihrem Äusserungskontext herausarbeiten lässt.

c) Die Autorschaft der Texte ist irrelevant für die Bedeutung der Texte.

3. Linguistik (und insbesondere die hermeneutische Korpuslinguistik) hat sich primär für die Bedeutung von Texten zu interessieren, sie soll diese herausarbeiten.

4. Der Begriff "Wort" ist zu vage und wird durch den Begriff Diskursobjekt abgelöst.

5. Das Diskursobjekt als sozial konstruierte Grösse bildet den zentralen Gegenstand der linguistischen Untersuchung.

6. Alles was im Diskurs über Diskursobjekte ausgesagt wird, gehört zu seiner Bedeutung.

7. Lexikalische Einheiten sind monoseme Bedeutungseinheiten in den Texten, sie können mehrere Wörter umfassen. Sie werden über Kollokationsanalysen ermittelt. Sie entsprechen oft den Diskursobjekten.

8. Die Bedeutung einer solchen Kollokation oder eines Diskursobjektes ist die von der Diskursgemeinschaft anerkannte Interpretation.

9. Die Interpretation von Texten geschieht vor dem Hintergrund des Diskurses, in dem der Text steht, und sie stellt primär die intertextuellen Bezüge her.

In dieser Replik werden einige Prämissen, Zielsetzungen und Konsequenzen der von Teubert vertretenen Position kritisch hinterfragt.

\section{Schriftlichkeit und Mündlichkeit}

Linguistik ist die Wissenschaft der menschlichen Sprachen, ihr Ziel ist es, die menschliche Sprachfähigkeit zu erforschen, sowie sämtliche kollektiven und individuellen Ausprägungen, die diese Fähigkeit hervorbringt. Sprache ist eine soziale Praxis, Sprache ist kein menschliches "Organ" oder "Modul", sondern ein emergentes Phänomen der sozialen Interaktion (Keller 1994), das auf zahlreiche neuronale, insbesondere sensorische und motorische Teilprozesse im menschlichen Körper zurückgreift. Die primäre phylogenetische und ontogenetische Form von Sprache ist die der Mündlichkeit. Von den schätzungsweise 7000 lebenden Sprachen (Gordon 2005) haben ungefähr 5\% mehr als eine Million SprecherInnen, die restlichen $95 \%$ der Sprachen sind kleine bis sehr kleine Sprachen. Nur ungefähr 6\% der Sprachen der Welt werden von 95\% der Weltbevölkerung gesprochen.

Kleine Sprachen verfügen oft über überhaupt keine Schriftlichkeit, oder diese ist im Alltag der SprecherInnen relativ marginal, wenn sie nicht gar in einer anderen, grösseren Sprache als der in der jeweiligen Kultur gesprochenen stattfindet. 
Eine Linguistik, die sich auf die Untersuchung geschriebener Texte beschränkt, klammert also a priori die Mehrheit der sprachlichen Systeme auf diesem Planeten aus. Sie beschränkt sich darüber hinaus aber auch im Bereich derjenigen (zumeist grossen) Sprachen, die eine ausgebaute Schriftlichkeit haben, auf die Untersuchung einer spezifischen Zustandsform, die quantitativ im Vergleich zur oralen Sprachproduktion und -rezeption marginal ist. Eine solche Selbstbeschränkung wäre fatal für den wissenschaftlichen Ruf unserer Disziplin. Es gibt meines Wissens auch keine BiologInnen, die fordern, ihre Disziplin solle sich ausschliesslich um Mikroben und Insekten befassen, weil diese den Grossteil der Biomasse ausmachen.

Die Aussage 1b), dass orale Kulturen keine metasprachlichen Diskurse kennen, ist reine Spekulation. Selbst ein kurzer Blick in gerade zur Verfügung stehende Arbeiten aus dem Bereich der anthropologischen Linguistik zeigt, dass orale Kulturen selbstverständlich eine grosse Vielfalt an metasprachlichen Diskursen aufweisen (z.B. Haviland 1979). In Kilivila (Papua Neu Guinea; vgl. Senft 2006) gibt es nicht nur Begriffe für 'Wort' und 'Satz', sondern die SprecherInnen unterscheiden auch eine Vielzahl von Dialekten sowie innerhalb einer lokalen Varietät jeweils einen zweidimensionalen Varietätenraum mit zwei generellen und sechs spezifischen Stilen, wobei generationelle, situationelle, wahrheitssemantische und weitere vor allem pragmatische Faktoren in die Kategorisierung einfliessen. Kilivila hat nicht nur einen Ausdruck für 'Wort' (kwetala biga), sondern auch eine Kategorie unterhalb der Morphemebene, für 'Laut' (luluvala).

In der Tat kennen viele Sprachen keinen Begriff, der mit dem westlichen linguistischen Wortbegriff genau synonym ist (Dixon/Aikehnvald 2002: 3), doch hat dies vielfältige Gründe, nicht zuletzt auch sprachtypologische. In einer polysynthetischen Sprache ist ein Wort beispielsweise eher eine komplexe syntaktische Einheit. In der chinesischen Linguistik gibt es zwar - entgegen gegenteiligen Beteuerungen - die Kategorie und den Begriff Wort, in der volkstümlichen Wahrnehmung jedoch dominiert der metasprachliche Begriff zì, der - es erstaunt sicherlich nicht - primär auf das Zeichen verweist (Dixon/Aikhenvald 2002: 33). Schrift und Schriftlichkeit ist also weder Voraussetzung noch Garant für eine Kategorie 'Wort', da diese schlicht stark an flektierende, vorwiegend synthetische westliche Sprachen gebunden ist. Fast alle Sprachen hingegen scheinen einen Begriff '(Eigen)Name' zu kennen (Dixon/Aikhenvald 2002: 3), der dann auch in ausweitendem Gebrauch als Wortbegriff verwendet wird - ein Bezug, der übrigens bereits im Kratylos-Dialog aufscheint. A propos Kratylos-Dialog: In diesem frühen Klassiker der westlichen Sprachreflexion führt Sokrates ein Gespräch mit den beiden Sophisten Kratylos und Hermogenes. Von Sokrates gibt es bekanntlich keinerlei schriftliche Hinterlassenschaft, seine Ideen wurden erst Jahrzehnte nach seinem Tod schriftlich festgehalten. Wer würde aber wagen, zu behaupten, Sokrates hätte in einem Umfeld gelebt, das nicht dazu in der Lage war, "vom Kontext losgelöste Diskursobjekte" zu schaffen?

Mündlichkeit geht in der Tat oft mit Kontextbezug, Deixis, situativ verknapptem Sprechen und vergleichsweise weniger Explizitheit einher (vgl. Koch/Oesterreicher 1985 oder 1994, vgl. auch Berthele 2006: 79ff.), doch gibt es auf der anderen Seite auch in mündlichen Gesellschaften Formen konzeptioneller Schriftlichkeit, die grosse Ähnlichkeiten mit der prototypi- 
schen Schriftlichkeit (Sprache der Distanz) aufweist ("elaborierte Mündlichkeit", vgl. Koch/Oesterreicher 1985: 30).

Mündlichkeit, Direktheit, Dialogizität ist die prototypische Zustandsform von Sprache. Eine Linguistik, die sich auf die abgeleitete Zustandsform der Schriftlichkeit beschränkt, hat empirisch Schlagseite und ist langfristig zum Sinken verdammt. Vom Kontext losgelöste Diskursobjekte (vgl. Punkt 1c) werden bereits in Kindergruppen geschaffen, deren Mitglieder im präoperationalen Stadium sind (Piaget/Inhelder 1986: 92). Bereits in dieser Phase verfügen Kinder über die Symbolfunktion und verstehen beispielsweise, dass eine Miniaturkonfiguration in einem Puppenhaus eine Konfiguration in der realen Welt repräsentieren kann. Das Bewusstsein der Symbolhaftigkeit und viele andere metalinguistische Fähigkeiten können bereits bei 3- bis 4-Jährigen nachgewiesen werden (Smith/Tager-Flusberg 1982), und besonders mehrsprachig aufwachsende Kinder (nach vielen Schätzungen die Mehrheit der Kinder auf dieser Welt, vgl. Cook 1993: 3) entwickeln sehr früh ein breites Repertoire von metalinguistischen Kenntnissen (Brédart/Rondal 1982; De Houwer 1995; Berthoud-Papandropoulou 1976). Metasprachliches Denken hat mit Spracherwerb und -verwendung im direkten, mündlichen Kontext zu tun, es setzt Schriftlichkeit in keiner Weise voraus. Dass letztere metasprachliche Diskurse stimulieren kann, ist dabei wohl unbestritten.

Wie das Beispiel der sokratisch-platonischen Dialoge sowie die Evidenz aus der anthropologischen Linguistik und aus der Spracherwerbsforschung klar zeigen, schafft Mündlichkeit nicht nur Diskursobjekte im Sinne dessen, was Teubert mit dem Begriff zu meinen scheint (genau definiert werden die Diskursobjekte nicht), sondern auch metasprachliche Diskurse auf bemerkenswertem Niveau. Dass diese oft nicht per Mausklick aus online Korpora extrahierbar, sondern nur durch Feldarbeit ermittelbar sind, heisst nicht, dass sie nicht existieren bzw. für die Linguistik nicht relevant wären.

\section{Worte, Diskursobjekte, Texte und Sprecherintentionen}

Ausgehend von seiner Charakterisierung der Mündlichkeit charakterisiert Teubert den schriftlichen Diskurs als Diskurs, der nicht (mehr) direkt auf sprachexterne Wirklichkeiten referiert, sondern primär auf sich selber verweist. Der schriftliche Diskurs schafft Objekte (Teubert nennt explizit Diskursobjekte wie Familie, Kindheit, Religion, Schule, Nation, Depression, Schönheit, Eigentum, Arbeit, Sprache). Dieses Potenzial, Diskursobjekte zu schaffen, ist gemäss Teubert eine genuine Eigenschaft der Schriftlichkeit. Es wurde am Beispiel Sprache oben schon gezeigt, dass zumindest dieses Diskursobjekt selbstverständlich auch ein Objekt mündlicher Kulturen sein kann. Nicht anders verhält es sich natürlich auch mit sozialen Institutionen wie Religion, Eigentum, Arbeit etc. Sicherlich verfügen so genannt "entwickelte" schriftliche Gesellschaften über eine beträchtliche Anzahl von einschlägigen Institutionen wie Schulen oder Ordnungsämtern, deren Existenz tatsächlich von und durch Schriftlichkeit geprägt ist. Zweifellos schafft die zeitliche und örtliche Distanz zwischen Produktion und Rezeption, welche die Schriftlichkeit erst ermöglicht, eine grössere Distanz zu den Referenten der Texte. Und diese grössere Distanz schlägt sich selbstverständlich in unterschiedlicher Ausgestaltung der sprachlichen Form nieder. Genau diese Eigenschaften 
sind auch seit längerem Gegenstand der einschlägigen Forschung zu Mündlichkeit und Schriftlichkeit (siehe oben), die m.E. bereits ihre Reflexe fand in den frühen soziolinguistischen Diskussionen um restringierten und elaborierten Code (Bernstein 1971), aber auch in der aus der funktionalistischen Linguistik stammenden Unterscheidung von pragmatischem und syntaktischem Modus (Givón 1979: 222). All dies ist unbestritten, bedeutet aber nicht, dass nicht auch in mündlichen Kulturen Diskursobjekte in einem diachronen Prozess geschaffen, tradiert, modifiziert und neu ausgehandelt werden.

Weder in der geschriebenen noch in der gesprochenen Sprache referieren Wörter, Texte oder Diskursobjekte an sich auf irgend etwas, sie tragen aber - zumindest in der Sichtweise der Semantik - eine vom Kontext relativ unabhängige Bedeutung (Denotat). Nicht Wörter oder Sätze, sondern einzig Äusserungen referieren (Lyons 1995: §7), also Wörter und Sätze, die von SprecherInnen im Kontext gebraucht werden. Die Semantik ist die Disziplin, welche die Bedeutung von Morphemen, Wörtern, Sätzen (und ev. Texten) losgelöst von ihren Äusserungskontexten untersucht. Sie interessiert sich allenfalls noch für das Potenzial, das es möglich macht, Ausdrücke in verschiedenen Äusserungskontexten mit sehr unterschiedlichen Bedeutungen zu verwenden. Die Pragmatik interessiert sich für die systematischen Zusammenhänge zwischen sprachlichen Formen, ihren BenutzerInnen und den konkreten Äusserungskontexten. Eine von Teubert postulierte hermeneutische Korpuslinguistik abstrahiert ganz bewusst von ProduzentInnen und RezipientInnen, reifiziert und agentiviert den Text, macht diesen zu einem Teil des "Diskurses". Die Bedeutung von Diskursobjekten sodann ergibt sich einzig aus der Analyse des Diskurses, dessen Paraphrasen die Bedeutungsexplikation darstellen. Man könnte also argumentieren, dass die hermeneutische Korpuslinguistik sich im Sinne der oben definierten Disziplin der Semantik für die Bedeutung und den Bedeutungswandel von Diskursobjekten interessiert. Es würde sich also um eine korpuslinguistisch basierte lexikalische Semantik handeln, wobei natürlich der Begriff der Lexik sehr weit gefasst wäre und Phraseologismen und Kollokationen mit einschliessen würde.

Teuberts Beispiele und die Abschnitte am Schluss seines Artikels machen dann jedoch klar, dass es nicht um eine solche textuell basierte Semantik geht, sondern um die Analyse von "Wirklichkeiten", er fordert eine Linguistik, die parallele, alternative Diskurse aufzeigt und vergleicht, um den Menschen einen "eigenen Standpunkt" zu ermöglichen. Alle Beispiele in Teuberts Text stammen denn auch aus dem Bereich der ideologischen und politischen Diskussionen um Krieg (friendly fire), Abtreibung (als Mord?), Migration (arrangierte Ehen, Zwangsehen), Europäische Union, Massenvernichtungswaffen. Zweifellos sind dies ergiebige Gegenstände der diachronen oder synchronen Semantik, es ist interessant, den Bedeutungswandel und Bezeichnungswandel in diesen Feldern systematisch aufzuarbeiten, und es ist absolut notwendig, hierfür ein adäquates Korpus zur Verfügung zu haben. Was aber in Anbetracht des differenzierten und elaborierten Instrumentariums der Linguistik erstaunt, ist der Versuch, hierfür eine eigene Disziplin der "hermeneutischen Korpuslinguistik" zu erschaffen, die den - zugegebenermassen nicht eindeutig definierten - Begriff Wort zugunsten des nicht besser definierten Begriffs Diskursobjekt abschaffen will, die zudem von den ProduzentInnen abstrahiert, wo es doch gerade in der Analyse der politischen Schimpf-, Schlag- und Fahnen- 
wörtern absolut essenziell ist, zu wissen, wer (zu wem) von Kapitalismus oder Neoliberalismus redet, wer sich selber oder andere als Nigger bezeichnet, etc. Auch schriftliche Texte werden in einem sprachlichen UND aussersprachlichen Kontext verfasst, und im Gegensatz zu Teuberts Grundannahme wird sogar im Falle der wohl explizitesten Textsorte, den Gesetzestexten, immer zu ergründen versucht, was die Intention der AutorInnen der Texte gewesen sein könnten - wie sonst könnte man auf der Basis von einem Urheberrechtsgesetz aus den 50er Jahren über Webinhalte und Neue Medien Recht sprechen, wie das in vielen Ländern noch immer gang und gäbe ist. Gerichtsurteile enthalten also nicht zufällig oft Verweise auf das, was der Gesetzgeber bei der Erarbeitung der betreffenden Paragraphen gemeint hatte.

Selbst im religiösen Bereich ist es eine Binsenwahrheit (zumindest in aufgeklärten Kreisen), dass die religiösen Texte nicht an sich etwas bedeuten, sondern nur adäquat rezipiert werden können, indem der aussersprachliche, kulturelle, eben lebensweltliche Kontext seiner Entstehungssituation rekonstruiert wird. Durch Rekonstruktion dieses Kontextes, zusammen mit der Rekonstruktion der möglichen Denotate der Wörter und Kollokationen, kann annäherungsweise erschlossen werden, was der Text bedeutete, worauf er referierte, was seine Produzenten intendierten, und inwiefern er heute relevant ist.

Teubert weist mit Recht darauf hin, dass es für den Begriff Wort in der Linguistik keine einheitliche, allen Bedürfnissen gerecht werdende Definition gibt. Trotzdem verwendet er genau wie alle übrigen LinguistInnen - den Begriff selber durchweg, und zwar sogar häufiger als den Begriff Diskursobjekt. Es gibt bekanntlich eine Vielzahl von Wortdefinitionen, die namentlich davon abhängen, welche linguistischen Fragen man beantworten will, welche Theorien man diesen Fragen zu Grunde legt, und welche Sprachen man betrachtet. Die Domäne dessen, was wir alltagssprachlich in ausreichender Präzision als "Wort" bezeichnen, ist durch linguistische Termini Lexem, Wortform, syntaktisches Wort, etc. ausreichend abgedeckt. Vagheit von Begriffen kann in multifaktoriellen Bereichen wie jenem der Sprachwissenschaft durchaus praktisch sein. Wie Teubert selber anführt, hat sich denn auch der Begriff des Wortes in der Linguistik durchaus als praktisch erwiesen. Und mehr als praktisch kann und soll ein Begriff oft auch gar nicht sein. Ob der Begriff des Diskursobjektes jedoch jemals so praktisch wird wie jener des Wortes, ist offen.

\section{Zum wissenschaftlichen Mehrwert der Hermeneutischen Korpuslinguistik}

Was also ist der Vorteil einer Disziplin, welche in ihrer Grundtendenz auf die theoretisch und empirisch bewährte Arbeitsteilung von Semantik und Pragmatik verzichtet (wobei eine solche Trennung natürlich niemals absolut sein kann), welche von den UrheberInnen und RezipientInnen von Wörtern, Sätzen und Texten abstrahiert, und welche den Text als Teil des Diskurses zum Akteur macht? Inwiefern können Fragen der politischen Bedeutungsverhandlung mit dieser neuen Disziplin besser analysiert werden als mit herkömmlichen Ansätzen? Und: Wenn auch niemand bestreitet, dass die Analyse politisch-ideologischer Diskurse ein möglicher und relevanter Spielplatz der Linguistik sein kann, worin liegt denn der spezifische Vorteil der "hermeneutischen Korpuslinguistik" für die Analyse der etwas weniger spektakulären lexikalischen Semantik der Präpositionen, Modalverben, Doppelperfektkonstruktionen, 
etc. - Oder sind dies alles keine lohnenswerten Unterfangen mehr? - Mir scheint, selbst wenn man das weite Feld der Semantik betrachtet, dass die Ziele und Methoden der in Teuberts Aufsatz postulierten Theorie extrem eng begrenzt sind. Teuberts eigener Text zeichnet sich denn auch durch einen Mangel an historischer Tiefe aus, obwohl er selbst seine Disziplin als diachron charakterisiert: Offensichtlich waren die befragten Korpora so eng gewählt, dass das Folgende behauptet werden kann:

Lexikalische Einheiten verändern ihre Bedeutung. Kein Mensch wäre vor fünfzig Jahren auf die Idee gekommen, das Diskursobjekt Abtreibung mit Mord an ungeborenen Kindern gleichzusetzen. (Teubert 2006: 57)

Kein Mensch - ausser den indischen Brahmanenphilosophen, einem gewissen Minucius Felix (3. Jh.), Basilius von Caesarea (im Jahr 374) und den Verfassern des Kanonischen Rechts der Katholischen Kirche (Canon 1398) - wie ein Blick in ein Konversationslexikon oder ein Klick in die Gratisenzyklopädie Wikipedia bereits nachweist. Für die diachrone Erforschung des Diskursobjekts Abtreibung ist Mord braucht es m.E. weder eine hermeneutische noch eine anderswie geartete Korpuslinguistik, und es braucht wohl überhaupt keine LinguistInnen, sondern gute HistorikerInnen.

Was also bringen Korpora uns LinguistInnen? Inwiefern verändern Korpora unsere Sicht auf mögliche Fragestellungen und deren Beantwortung? Welche Korpora brauchen wir zusätzlich $\mathrm{zu}$ denen, die es schon gibt? - Dies wäre natürlich Gegenstand für einen eigenständigen Beitrag, diese Fragen können hier nicht abschliessend beantwortet werden. Da aber Teuberts Vorschlag m.E. etwas einseitig und für das, was wir traditionellerweise unter den Zielen der Linguistik verstehen, nur teilweise ergiebig ist, sollen Alternativen hier abschliessend zumindest stichwortartig benannt werden.

\section{$5 \quad$ Was bringt Korpuslinguistik der Linguistik? - Eine unvollständige Liste}

Linguistik ist die Wissenschaft von Sprache. Sprache ist eine soziale Praxis, die sich mündlich und/oder schriftlich manifestieren kann. Die Disziplin der Linguistik evoluierte von Beginn weg im Spannungsfeld deduktiv orientierter Theoriebildung und datengeleiteter Induktion. Um eine empirische Absicherung kommen inzwischen nicht einmal mehr die eingefleischtesten Generativen GrammatikerInnen herum. Empirie kann Arbeit mit Experimenten, mit spontansprachlichen Korpora, mit gezielt elizitierten Korpora und in Ausnahmefällen auch noch immer mit Rückgriff auf Kompetenzbelege heissen. Sozialwissenschaftlich und psychologisch orientierte LinguistInnen stellen Hypothesen auf, die sie dann empirisch zu überprüfen versuchen, um die Theorien, von denen sie ausgegangen sind, zu revidieren oder gar ganz abzulehnen.

Korpuslinguistik ist also keine Theorie, sondern eine Methode, und zwar eine exzellente Methode, die helfen kann 
die lexikalische Semantik von Einheiten unterschiedlicher Komplexität (Lexeme, Phraseologismen, Kollokationen) zu beschreiben

variationistische Analysen von stilistischen, arealen, dialektalen Phänomenen durchzuführen

empirisch valide Wörterbücher zu verfassen

Bedeutungswandel zu beschreiben

Kollokationen und idiomatische Fügungen zu ermitteln und beschreiben

Konstruktionen im (grossen) Grenzbereich von Lexikon und Syntax herauszuarbeiten (vgl. die vielversprechenden Ansätze im Umfeld der Collostructional Analysis, z.B. Gries/Stefanowitsch 2004)

Häufigkeiten von Satzmodellen zu ermitteln (über tief annotierte Korpora; vgl. TIGER), um z.B. auch den Fremdsprachenunterricht effizienter zu gestalten

Das von Teubert skizzierte Programm jedoch käme einer Linguistik gleich, die sich ohne Not empirisch, thematisch und theoretisch extrem einengt. Gleichzeitig würden Begriffe und Methoden der empirischen Arbeit mit Sprachdaten aufgegeben, die sich in den letzten Jahrzehnten einen gewissen intra- und interdisziplinären Respekt verschafft haben. Die "hermeneutische Korpuslinguistik" erscheint als eine neue Form der Lehnstuhllinguistik, die so konstruiert ist, dass genau die Daten abgefragt werden können, die von ein paar wenigen Sprachen in ganz gewissen sprachlichen Lekten und Registern massenhaft und in der Regel online verfügbar sind. Sicher bringt die Möglichkeit der Analyse von Chat-Daten und anderen informelleren Registern auch einen gewissen Grad von kolloquialem und konzeptionell mündlichem Schreiben in Reichweite einer solchen Korpusanalyse. Doch trotz alledem bleibt die Anzahl der Sprachen und Lekte stark auf den westlichen Kontext eingeengt und begrenzt. Es soll hier also keineswegs bestritten werden, dass wir heute über gewisse Register und Sprachen auf der Basis der verfügbaren Korpora neue und starke Aussagen machen können auch bezüglich der Semantik von lexikalischen Einheiten. Dabei handelt es sich um Aussagen, die zuvor tatsächlich in dieser empirisch abgesicherten Form nicht möglich gewesen wären. Das ist aber nur ein kleiner Teil der Linguistik. Korpora von europäischen Standardsprachen sind eine tolle Sache, doch bis auf Weiteres kommt eine Linguistik, die nach möglichst generalisierbaren Beschreibungen von Phonologie, Syntax, Morphologie, Semantik und Pragmatik von Sprachen in ihrer typologischen, diachronen und diastratischen Vielfalt strebt, nicht um einen Methodenpluralismus herum. Feldarbeit und empirische Forschungsdesigns, gerade auch zur Erforschung der zahlreichen primär oder exklusiv mündlichen Sprachen, Dialekte und Register, werden auch weiterhin mit den Wort- und Kollokationssuchen in schriftsprachlichen Korpora koexistieren. Die moderne Linguistik tut zweifellos gut daran, sich auf die interdisziplinäre Einbettung ihrer Modelle in Modelle der Psychologie und Soziologie - letzteres scheint letztlich Teuberts (m.E. sehr lobenswerte) Intention und Ambition zu sein - zu besinnen. Gleichzeitig sollte sie aber auch nicht vorschnell die genuin linguistischen Kategorien, Differenzierungen und disziplinären Ambitionen zugunsten von Anleihen bei Schleiermacher und Luhmann aufgeben. 


\section{Literaturangaben}

Bernstein, Basil (1971): Class, Codes and Control (Volume 1). London.

Berthele, Raphael (2006): Ort und Weg. Eine vergleichende Untersuchung der sprachlichen Raumreferenz in Varietäten des Deutschen, Rätoromanischen und Französischen. Berlin/New York.

Berthoud-Papandropoulou, Ioanna (1976): La réflexion metalinguistique chez l'enfant. Genève.

Brédart, Serge/Rondal, Jean-Adolphe (1982): L'analyse du langage chez l'enfant: les activités métalinguistiques. Bruxelles.

Cook, Vivian J. (1993): "Wholistic multi-competence - jeu d'esprit or paradigm shift?". In: Kettemann, Bernhard/Wieden, Wilfried (eds.): Current Issues in European Second Language Acquisition Research. Tübingen: 3-9.

De Houwer, Annick (1995): "Bilingual Language Acquisition". In: Fletcher, Paul/MacWhinney, Brian (eds.): The handbook of child language. Oxford/Cambridge Mass.: 219-250.

Dixon, R. M. W./Aikhenvald, Alexandra (2002): Word: A cross-linguistic typology. Cambridge, UK.

Givón, Talmy (1979): On understanding Grammar. New York.

Gordon, Raymond G. (ed.) (2005): Ethnologue: Languages of the World. Dallas, TX.

Gries, Stefan T./Stefanowitsch, Anatol (2004): "Extending collostructional analysis: a corpusbased perspective on 'alternations'". International Journal of Corpus Linguistics 9, 1: 97-129.

Haviland, John (1979): "How to Talk to Your Brother-in-Law in Guugu Yimidhirr". In: Shopen, Timothy (ed.): Languages and Their Speakers. Cambridge, Massachusetts: 161240.

Keller, Rudi (1994): Sprachwandel. Von der unsichtbaren Hand in der Sprache. Tübingen/Basel.

Koch, Peter/Oesterreicher, Wulf (1985): "Sprache der Nähe - Sprache der Distanz: Mündlichkeit und Schriftlichkeit im Spannungsfeld von Sprachtheorie und Sprachgeschichte". Romanistisches Jahrbuch 36: 15-43.

Koch, Peter/Oesterreicher, Wulf (1994): "Funktionale Aspekte der Schriftkultur. Schriftlichkeit und Sprache". In: Günther, Hartmut/Ludwig, Otto (eds.): Schrift und Schriftlichkeit: ein interdiziplinäres Handbuch internationaler Forschung/Writing and its use: an interdisciplinary handbook of international research. Zusammen mit Jürgen Baurmann et al. Berlin/New York: 587-604.

Lyons, John (1995): Linguistic Semantics. An Introduction. Cambridge.

Piaget, Jean/Inhelder, Bärbel (1986): Die Psychologie des Kindes. München.

Senft, Gunter (2006): Metalinguistic Terms in Kilivila - a preliminary overview. Manuskript: MPI Nijmegen.

Smith, C. L./Tager-Flusberg, Helen (1982): "Metalinguistic awareness and language development". Journal of Experimental Child Psychology 34: 449-468. 
Teubert, Wolfgang (2006): "Korpuslinguistik, Hermeneutik und die soziale Konstruktion der Wirklichkeit". Linguistik online 28, 3/06: 41-60. 